JOURNAL of

MAINE MEDICAL CENTER Journal of Maine Medical Center

Volume 4

Issue 1 Winter 2022

Article 7

2022

\title{
Telemedicine Consultation to Assess Neonatal Encephalopathy in Rural Community Hospitals and Tertiary Care Centers
}

\author{
Rachel Coffey \\ Maine Medical Center
}

Et al.

Follow this and additional works at: https://knowledgeconnection.mainehealth.org/jmmc

Part of the Congenital, Hereditary, and Neonatal Diseases and Abnormalities Commons, Neurology Commons, Pediatrics Commons, and the Telemedicine Commons

\section{Recommended Citation}

Coffey, Rachel; Melendi, Misty; Cutler, Anya K.; and Craig, Alexa K. (2022) "Telemedicine Consultation to Assess Neonatal Encephalopathy in Rural Community Hospitals and Tertiary Care Centers," Journal of Maine Medical Center. Vol. 4 : Iss. 1 , Article 7.

Available at: https://knowledgeconnection.mainehealth.org/jmmc/vol4/iss1/7 https://doi.org/10.46804/

2641-2225.1115

The views and thoughts expressed in this manuscript belong solely to the author[s] and do not reflect the opinions of the Journal of Maine Medical Center or MaineHealth.

This Innovation Highlight is brought to you for free and open access by Maine Medical Center Department of Medical Education. It has been accepted for inclusion in the Journal of Maine Medical Center by an authorized editor of the MaineHealth Knowledge Connection. For more information, please contact Dina McKelvy mckeld1@mmc.org.

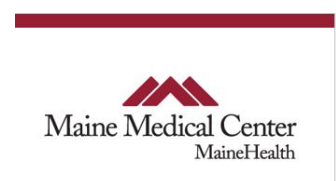




\section{Telemedicine Consultation to Assess Neonatal Encephalopathy in Rural Community Hospitals and Tertiary Care Centers}

\section{Authors}

Rachel Coffey, Misty Melendi, Anya K. Cutler, and Alexa K. Craig 


\title{
Telemedicine Consultation to Assess Neonatal Encephalopathy in Rural Community Hospitals and Tertiary Care Centers
}

\author{
Rachel Coffey, DO, ${ }^{1}$ Misty Melendi, MD, ${ }^{1,2}$ Anya K Cutler, MS, MPH, ${ }^{3}$ Alexa K Craig, MD, MS ${ }^{1,4}$ \\ ${ }^{1}$ Maine Medical Center, Department of Pediatrics, Barbara Bush Children's Hospital, Portland, ME, 'Maine Medical Center, \\ Division of Neonatology, Barbara Bush Children's Hospital, Portland, ME, 'Maine Medical Center, Center for Outcomes \\ Research, Portland, ME, “Maine Medical Center, Division of Pediatric Neurology, Barbara Bush Children's Hospital, Portland, \\ $M E$
}

Introduction:

Teleconsultation is used in tertiary care hospitals to evaluate neonatal encephalopathy. Neonates born in community hospitals, however, often experience delayed evaluation due to transport to the tertiary care center. We studied teleconsultations in community hospitals to decrease this disparity.

Methods: $\quad$ Prospective observational study in 9 community hospitals and 1 neonatal intensive care unit. Inclusion criteria: gestational age greater than or equal to 35 weeks and one of the following: umbilical cord $\mathrm{pH}$ less than or equal to 7.2,5-minute Apgar less than 7, prolonged respiratory support, perinatal event, or abnormal neurological exam. We performed synchronized, unscheduled telemedicine consults with the main outcome of time to teleconsultation.

Results: $\quad$ From April 2018 to September 2020, we performed 53 teleconsultations: 34 (64\%) in community hospitals and $19(36 \%)$ in the tertiary care center. Teleconsultations occurred at a median of 98 minutes (IQR, 76-127) in community hospitals versus 68 minutes (IQR, 43-91) in the tertiary care center $(P=$ $.004)$. Nine (26\%) neonates born in a community hospital remained with their parents and were not transferred to the tertiary care center for further assessment.

Discussion: Neonates born in rural community hospitals have slightly later teleconsultations than neonates born in the tertiary care center. Telemedicine use reduced this disparity from nearly 5 hours in our prior study to 98 minutes in this study by permitting evaluation of neonates in community hospitals without transporting them to the tertiary care center.

Conclusions: Teleconsultations to evaluate neonatal encephalopathy are a feasible, accessible, and reliable way to bring expert-level care into rural community hospitals.

Keywords: neonatal encephalopathy, therapeutic hypothermia, telemedicine, health care disparity, rural hospitals

$\mathrm{N}$ eonatal encephalopathy (NE) is a clinical syndrome of abnormal neurological function in a neonate at greater than 35 weeks gestation. ${ }^{1}$ Symptoms of NE can include abnormalities in any of the following: level of consciousness, spontaneous movement, muscle tone, posture, primitive reflexes (e.g., Moro and suck), and autonomic functions (e.g., heart rate, respiration, pupil function). ${ }^{2,3}$ Neonates with

Correspondence: Alexa K. Craig, MD

Maine Medical Partners Pediatric Neurology

92 Campus Drive, Scarborough, Maine, 04074

craiga@mmc.org moderate to severe $\mathrm{NE}$, acidotic umbilical cord $\mathrm{pH}$, and low Apgar scores are eligible for therapeutic hypothermia $(\mathrm{TH}),{ }^{4}$ a neuroprotective therapy that must be started in the first 6 hours of life for maximal effect. ${ }^{5} \mathrm{NE}$ can fluctuate within the first several hours of life,${ }^{6}$ highlighting the importance of experienced provider assessments (e.g., pediatric neurologist, neonatologist) and access to serial examinations. ${ }^{7}$

Newborns who are delivered in small community hospitals and may be eligible for $\mathrm{TH}$ need to be transferred to tertiary care centers for evaluation. 
This transfer can complicate the timeliness of assessment and initiation of therapy. ${ }^{8}$ Telemedicine use is rapidly expanding for multiple indications in the neonatal intensive care unit, ${ }^{9,10}$ especially for neurological indications given the high reproducibility of neonatal neurological exams performed by telemedicine consults. ${ }^{11} \mathrm{NE}$ assessment by telemedicine within the tertiary care facility is feasible. Unfortunately, for neonates born in community hospitals, the time required for transfer to a tertiary care center can delay this evaluation by hours. ${ }^{12}$

To assess feasibility of telemedicine use in the nontertiary care setting, we implemented synchronous, unscheduled telemedicine consults for neonates with suspected symptoms of NE in the community hospital. Our primary outcome measure was the time to teleconsultation, measured in hours from birth.

\section{METHODS}

\section{Study design}

Prospectively recruited neonates who received telemedicine consults from April 2018 to September 2020. The MaineHealth Institutional Review Board approved this study (\#1173919).

\section{Inclusion/Exclusion criteria}

Neonates were included if their gestational age was greater than or equal to 35 weeks and they showed one of the following signs of a difficult delivery: cord $\mathrm{pH}$ less than or equal to 7.2, 5-minute Apgar less than 7, prolonged respiratory support, perinatal event (e.g., dystocia, cord prolapse, placental abruption, uterine rupture), or abnormal neurological exam. Infants more than 6 hours old at the time of telemedicine consultation were excluded.

\section{Teleconsultation}

In the tertiary care center, we used medical grade, wheeled telemedicine carts with a 32-inch screen and a high-resolution, zoom-capable camera with Cisco Jabber video conferencing software for a two-way interface between the pediatric neurologist and neonatologist. In the community hospital, we used Vidyo® software, which was capable of a three-way interface between the pediatric neurologist, the neonatologist, and the community hospital pediatrician. Both software platforms were https://knowledgeconnection.mainehealth.org/jmmc/vol4/iss 1/7 encrypted and compliant with the Health Insurance Portability and Accountability Act. The telemedicine carts were plugged into the Ethernet to ensure high-quality audio/video feeds.

\section{Neonatal encephalopathy exam}

We examined neonates using a modified version of the Sarnat staging exam. ${ }^{2,4}$ This exam involves categorizing the level of consciousness, spontaneous movements, muscle tone, distal flexion, quality of the suck reflex, Moro reflex, and autonomic features, including heart rate, respiratory rate, and pupillary function (Supplement 1). We assigned scores of 1 for mildly abnormal features, 2 for moderately abnormal features, and 3 for severely abnormal features. Individual scores were added for a total score. The neurologist assigned the NE score by observing the neonate through the telemedicine consult. Scores ranged from 1 to 6 for mild NE, 7 to 18 for moderate NE, and 19 to 27 for severe NE. As NE can be a waxing and waning clinical condition, we repeated telemedicine exams for neonates who did not meet inclusion criteria for treatment with $\mathrm{TH}$ as long as the exam could be started within the 6-hour window.

\section{Statistical analysis}

Patients were grouped by birth location (community hospital vs tertiary care center), and statistical differences were determined for several clinical characteristics. Student's $t$ tests were used for normally distributed continuous variables, and Wilcoxon rank-sum tests were used for nonnormally distributed variables. Normality was determined by visualizing histograms and Shapiro tests. Chi-squared tests were used for categorical variables, unless expected cell counts were less than 5, in which case Fisher's exact tests were used. All analyses were performed in R 3.6.2.

\section{RESULTS}

From April 4, 2018, to September 24, 2020, there were 57 neonates who received telemedicine consults to evaluate for NE. Four neonates were excluded: 1 who was born at the tertiary care center at a gestational age less than 35 weeks, and 3 who were born at community hospitals and the teleconsultation was requested after the neonate was transferred to the tertiary care center for other reasons (e.g., respiratory distress). Nineteen (36\%) neonates were born in the tertiary care center, and the remaining $34(64 \%)$ were born in community 
hospitals. Neonates from the tertiary care center were born at significantly younger gestational ages, had smaller birth weights, and were delivered via cesarean section at a higher rate than neonates born in community hospitals (Table 1). At the tertiary care center, patients' umbilical cord gases had lower $\mathrm{pH}$ and higher base deficits than patients at community hospitals. Twenty-four $(45 \%)$ neonates were treated with $\mathrm{TH}$. Of these, $7(37 \%)$ were born in the tertiary care center and $17(50 \%)$ were born in community hospitals.
The first teleconsultation occurred at a median of 98 minutes (IQR, 76-127) in community hospitals compared to 66 minutes (IQR, 43-91) in tertiary care centers $(P=.004)$ (Table 2$)$. More neonates at the tertiary care center $(n=14,74 \%)$ had second telemedicine consults than neonates born at community hospitals $(n=12,35 \%)$. For the neonates who received second and third consults, the interval time between those assessments was not different between cohorts. After teleconsultation, $9(26 \%)$ neonates born at community hospitals were not transferred to the tertiary care center for

Table 1: Table 1. Maternal and Neonatal Characteristics

\begin{tabular}{|c|c|c|c|}
\hline Characteristic & $\begin{array}{l}\text { Tertiary Care Center, } \\
N=19^{1}\end{array}$ & $\begin{array}{l}\text { Community } \\
\mathrm{N}=34^{1}\end{array}$ & Hospitals, $p$-value \\
\hline Maternal Age & $30(6)$ & $30(5)$ & 0.789 \\
\hline Unknown & 0 & 4 & \\
\hline Gestational Diabetes & $4(21 \%)$ & $4(12 \%)$ & 0.436 \\
\hline GBS Positive & $5(26 \%)$ & $6(18 \%)$ & 0.496 \\
\hline Preeclampsia/Eclampsia & $2(11 \%)$ & $2(5.9 \%)$ & 0.612 \\
\hline Prolonged Rupture of Membranes & $2(11 \%)$ & $9(26 \%)$ & 0.290 \\
\hline C-Section & $13(68 \%)$ & $10(29 \%)$ & 0.006 \\
\hline Gestational Age 35-36 wks & $4(21 \%)$ & $1(2.9 \%)$ & 0.050 \\
\hline Male Sex & $11(58 \%)$ & $24(71 \%)$ & 0.349 \\
\hline Birth Weight (kg) & $3.17(0.53)$ & $3.64(0.52)$ & 0.004 \\
\hline Unknown & 0 & 3 & \\
\hline APGAR at 1 Minute & $3(2)$ & $2(2)$ & 0.595 \\
\hline APGAR at 5 Minutes & $7(2)$ & $5(2)$ & 0.016 \\
\hline APGAR at 10 Minutes & $7(1)$ & $6(2)$ & 0.436 \\
\hline Unknown & 11 & 7 & \\
\hline Umbilical Cord ABG/VBG Obtained & $18(95 \%)$ & $26(76 \%)$ & 0.133 \\
\hline Arterial Cord pH & $7.07(0.16)$ & $7.15(0.14)$ & 0.108 \\
\hline Unknown & 2 & 10 & \\
\hline Arterial Base Deficit & $-13.6(7.4)$ & $-10.0(4.9)$ & 0.085 \\
\hline Unknown & 2 & 12 & \\
\hline Venous Cord pH & $7.14(0.14)$ & $7.20(0.11)$ & 0.151 \\
\hline Unknown & 2 & 11 & \\
\hline Venous Base Deficit & $-10.9(5.9)$ & $-8.6(4.5)$ & 0.280 \\
\hline Unknown & 2 & 12 & \\
\hline $\begin{array}{l}\text { Infant ABG or VBG or capillary blood gas } \\
\text { within First Hour of Life }\end{array}$ & $10(53 \%)$ & $16(48 \%)$ & 0.773 \\
\hline Unknown & 0 & 1 & \\
\hline $\mathrm{pH}$ & $7.25(0.09)$ & $7.17(0.16)$ & 0.139 \\
\hline Unknown & 9 & 18 & \\
\hline Base Deficit & $-11.5(5.3)$ & $-10.8(5.9)$ & 0.763 \\
\hline Unknown & 9 & 20 & \\
\hline Received Therapeutic Hypothermia & $7(37 \%)$ & $17(50 \%)$ & 0.356 \\
\hline
\end{tabular}

Abbreviations: ABG, arterial blood gases; GBS, group B strep; SD standard deviation; VBG venous blood gases

${ }^{1}$ Mean (SD); n (\%)

${ }^{2}$ Wilcoxon rank sum test; Fisher's exact test; Pearson's Chi-squared test; Welch Two Sample t-test 
Table 2. Encephalopathy Scores and Timing of Scores

\begin{tabular}{|c|c|c|c|}
\hline Characteristic & $\begin{array}{l}\text { Tertiary care center } \\
(n=19)\end{array}$ & $\begin{array}{l}\text { Community hospitals } \\
(n=34)\end{array}$ & $P$ value $^{1}$ \\
\hline \multicolumn{4}{|l|}{ First encephalopathy score } \\
\hline No. & 19 & 34 & 0.422 \\
\hline Median (IQR) & $4(2,6)$ & $6(1,9)$ & \\
\hline Time from birth to first consult (min) & $66(43,91)$ & $98(76,127)$ & 0.004 \\
\hline \multicolumn{4}{|l|}{ Second encephalopathy score } \\
\hline No. & 14 & 12 & 0.513 \\
\hline Median (IQR) & $2(0,4)$ & $4(0,8)$ & \\
\hline Time from first to second consult (min) & $106(94,132)$ & $151(103,194)$ & 0.292 \\
\hline \multicolumn{4}{|l|}{ Third encephalopathy score } \\
\hline No. & 4 & 2 & 0.100 \\
\hline Median (IQR) & $0(0,2)$ & $9(8,10)$ & \\
\hline Time from second to third consult (min) & $130(116,146)$ & $108(99,116)$ & 0.533 \\
\hline
\end{tabular}

Abbreviations: IQR, interquartile range. ${ }^{1}$ Wilcoxon rank sum test

further medical assessment or management. Of the 25 neonates who were born at community hospitals and transferred, 8 (33\%) were ultimately not treated with $\mathrm{TH}$ despite being transferred.

\section{DISCUSSION}

This study demonstrates that teleconsultations for evaluating NE are a feasible, accessible, and reliable way to bring expert-level care into rural community hospitals to evaluate neonates who may be eligible for $\mathrm{TH}$. Although neonates born in community hospitals still have later teleconsultations than those born in the tertiary care center, the disparity decreased from nearly 5 hours (when the teleconsultation occurred after transfer to the tertiary care center ${ }^{12}$ ) to approximately 1.5 hours (when teleconsultation occurred at the rural community hospital). We show that the highresolution, zoom capable camera controlled by the neurologist from a remote location was an interface that worked successfully to assess neonates born in community hospitals. The telemedicine cart was set up with a hard-wired device (plugged into an Ethernet socket) to avoid complications with video and audio quality related to low bandwidth from wireless connections that previously compromised user satisfaction. ${ }^{10}$

Telemedicine evaluation for genetic and neurological examination accurately and reliably identifies exam abnormalities when compared to the gold standard, https://knowledgeconnection.mainehealth.org/jmmc/vol4/iss $1 / 7$ a bedside examiner. ${ }^{11}$ Our prior work showed that telemedicine assessments could be performed within the tertiary care center to specifically evaluate NE. ${ }^{12}$ This study highlights how telemedicine can improve access to repeated neurological exams for neonates born in either the tertiary care center or a community hospital. Almost $75 \%$ of neonates born in the tertiary care center and $35 \%$ of those born in a community hospital had access to a second neurological assessment in the first 3 hours of life. With the increasing complexity of decision-making surrounding the use of $\mathrm{TH}$ for symptoms of mild $\mathrm{NE},{ }^{13}$ serial neurological examinations ${ }^{7}$ of neonates by telemedicine may, at minimum, offer clinicians more information on which to base their clinical decisions within the critical 6- hour timeframe.

Our study also shows that using telemedicine consults in rural community hospitals prevented the transfer of approximately one quarter of the neonates assessed. In this project, the research funds were used to develop the infrastructure of the camera system in multiple community hospitals. Thus, we were unable to obtain brain imaging or neurodevelopmental outcome data on patients who were excluded from $\mathrm{TH}$ to be certain the correct clinical decision was made. We are careful to only claim that the telemedicine consultation may have helped to avoid unnecessary transfer, not that the clinical decision-making was correct. Avoiding unnecessary neonatal transports is desirable to 
prevent unnecessary separation of families ${ }^{14}$ and excessive cost of transport and admission to the neonatal intensive care unit. In rural states with limited resources, transport team arrival could be largely influenced by retrieval destination and availability of transport resources (e.g., staffing, proper equipment, ambulance). Other investigators have reported on the successful use of telemedicine consults to decrease neonatal transport. ${ }^{15,16}$

A strength of this study is that the telemedicine technology was applied in a relatively large network of 9 community hospitals in the most rural state in the United States. ${ }^{17}$

The main limitations of this study include the lack of outcome data for neonates that were not treated with $\mathrm{TH}$, the absence of data assessing how the teleconsultation affects transfer times, and an acknowledgment that these results reflect the experience of 1 telemedicine network and may not be generalizable to other health care systems. Future research will study short-term outcomes of electroencephalogram and magnetic resonance imaging on neonates excluded from $\mathrm{TH}$ and longterm neurodevelopmental outcomes to further verify the clinical decisions made by teleconsultation.

\section{CONCLUSIONS}

In summary, our study shows that teleconsultations are a feasible, accessible, and reliable approach to evaluating NE and triaging neonates in community hospitals. Our study also highlights how telemedicine can improve access to repeated neurological exams for neonates born in tertiary care centers or community hospitals. These findings support efforts that may lead to more efficient care for neonates in a resource-scarce environment.

\section{Conflicts of interest None}

\section{Acknowledgments}

We thank the families who chose to participate in this study for their generosity. We also thank the primary care physicians from the community hospitals for their participation.

Funding: This research was supported by grant U54 GM115516 from the National Institutes of Health for the Northern New England Clinical and Translational Research network.

\section{REFERENCES}

1. Executive summary: Neonatal encephalopathy and neurologic outcome, second edition. Report of the American College of Obstetricians and Gynecologists' Task Force on Neonatal Encephalopathy. Obstet Gynecol. 2014;123(4):896-901. doi:10.1097/01.AOG.0000445580.65983.d2

2. Sarnat HB, Sarnat MS. Neonatal encephalopathy following fetal distress. A clinical and electroencephalographic study. Arch Neurol. 1976;33(10):696-705. doi:10.1001/ archneur.1976.00500100030012

3. Volpe JJ. Neurology of the newborn. 5th ed. Saunders/Elsevier; 2008.

4. Jacobs SE, Berg M, Hunt R, Tarnow-Mordi WO, Inder TE, Davis PG. Cooling for newborns with hypoxic ischaemic encephalopathy. Cochrane Database Syst Rev. 2013;2013(1):CD003311. doi:10.1002/14651858.CD003311.pub3

5. Laptook AR, Shankaran S, Tyson JE, et al. Effect of therapeutic hypothermia initiated after 6 hours of age on death or disability among newborns with hypoxic-ischemic encephalopathy: a randomized clinical trial. JAMA. 2017;318(16):1550-1560. doi:10.1001/jama.2017.14972

6. Lally PJ, Montaldo P, Oliveira V, et al. Residual brain injury after early discontinuation of cooling therapy in mild neonatal encephalopathy. Arch Dis Child Fetal Neonatal Ed. 2018;103(4):F383-F387. doi:10.1136/archdischild-2017-313321

7. Natarajan G, Laptook A, Shankaran S. Therapeutic hypothermia: how can we optimize this therapy to further improve outcomes? Clin Perinatol. 2018;45(2):241- 255. doi:10.1016/j. clp.2018.01.010

8. Roberts CT, Stewart MJ, Jacobs SE. Earlier initiation of therapeutic hypothermia by non-tertiary neonatal units in Victoria, Australia. Neonatology. 2016;110(1):33-39. doi:10.1159/000444274

9. Lapcharoensap W, Lund K, Huynh T. Telemedicine in neonatal medicine and resuscitation. Curr Opin Pediatr. 2021;33(2):203208. doi:10.1097/MOP.0000000000000995

10. Fang JL, Collura CA, Johnson RV, et al. Emergency video telemedicine consultation for newborn resuscitations: the Mayo Clinic experience. Mayo Clin Proc. 2016;91(12):1735-1743. doi:10.1016/j.mayocp.2016.08.006

11. Wenger TL, Gerdes J, Taub K, Swarr DT, Deardorff MA, Abend NS. Telemedicine for genetic and neurologic evaluation in the neonatal intensive care unit. $J$ Perinatol. 2014;34(3):234-240. doi:10.1038/jp.2013.159

12. Craig AK, McAllister LM, Evans S, Melendi ME. Telemedicine consults to assess neonatal encephalopathy are feasible in the neonatal intensive care unit. $J$ Perinatol. 2020;41(6):1519-1521. doi:10.1038/s41372-020-00828-3

13. Burnsed J, Zanelli SA. Neonatal therapeutic hypothermia outside of standard guidelines: a survey of U.S. neonatologists. Acta Paediatr. 2017;106(11):1772- 1779. doi:10.1111/apa.13983

14. Craig AK, James C, Bainter J, Evans S, Gerwin R. Parental perceptions of neonatal therapeutic hypothermia; emotional and healing experiences. J Matern Fetal Neonatal Med. 2020;33(17):2889-2896. doi:10.1080/14767058.2018.1563592

15. Albritton J, Maddox L, Dalto J, Ridout E, Minton S. The effect of a newborn telehealth program on transfers avoided: a multiplebaseline study. Health Aff (Millwood). 2018;37(12):1990-1996. doi:10.1377/hlthaff.2018.05133

16. Armfield NR, Donovan T, Bensink ME, Smith AC. The costs and potential savings of telemedicine for acute care neonatal consultation: preliminary findings. $J$ Telemed Telecare. 2012;18(8):429-433. doi:10.1258/jtt.2012.gth10 